\title{
Évaluation des modes de déplacement d'un robot à locomotion hybride roue-patte évoluant en milieux naturels
}

\author{
Christophe Granda ${ }^{a}$ Faïz Ben Amar, Frédéric Plumet et Philippe Bidaud \\ Laboratoire de Robotique de Paris, Université de Paris 6, CNRS FRE2507, 18 rue du Panorama, BP 61, \\ 92265 Fontenay-aux-Roses Cedex, France
}

Reçu le 25 février 2005, accepté le 21 mars 2005

\begin{abstract}
Résumé - Cet article s'intéresse au problème de l'évaluation des modes de déplacement d'un robot à locomotion hybride roue-patte. Après une brève description du système mécatronique, nous présentons sa modélisation cinémato-statique dont l'originalité repose sur l'intégration des interactions roue-sol en terrains naturels. Les modèles d'interaction sont validés expérimentalement à l'aide d'un banc d'essai spécifique. Les modèles analytiques sont ensuite exploités pour réaliser une analyse comparative des performances de locomotion pour chacun des modes de déplacement sur différents terrains. Les critères considérés sont la marge de stabilité, la gradabilité et la consommation d'énergie.
\end{abstract}

Mots clés : Robot mobile / hybride / roue-patte / locomotion / modélisation / performances / terrains naturels / terra-mécanique / expérimental

\begin{abstract}
Evaluation of the locomotion modes for an hybrid wheel-legged robot evolving in outdoor environment. This article deals with the problem of locomotion modes evaluation of an hybrid wheel-legged robot. After a brief mechatronic description of the system, we present its kinetostatic modeling whose originality is the integration of the wheel-ground interaction models on natural terrains. These interaction models are experimentally validated on a specific test-bed. Then, the analytical models are exploited to carry out a comparative analysis of the performances of locomotion for each mode on different terrains. The considered criteria are the stability margin, the gradability and the energy consumption.
\end{abstract}

Key words: Mobile robot / hybrid / wheel-legged / locomotion / modeling / performance / natural terrains / terramechanics / experimental

\section{Introduction}

Les robots mobiles tout terrain trouvent leurs applications de nos jours dans plusieurs domaines. On identifie principalement l'exploration terrestre (polaire et volcanologique) ou planétaire (lune, Mars) ainsi que les applications militaires ou civiles pour l'observation et la surveillance dans des environnements naturels. Les sols sur lesquels doivent évoluer ces engins présentent par endroit des difficultés de franchissement majeures qui se traduisent à la fois en terme de géométrie de la surface (discontinuités, relief, densité de roches ...) et de nature physique du sol (sol meuble, sol non-cohésif, sable, éboulis, ....).

L'introduction des mobilités internes dans la structure cinématique de l'appareil locomoteur des robots d'exploration répond à ce besoin d'une mobilité et d'une

${ }^{\text {a }}$ Auteur correspondant : grand@robot.jussieu.fr capacité de franchissement accrue. Nous pensons, en effet, qu'un des moyens permettant d'augmenter l'autonomie de déplacement d'un robot en milieu naturel ouvert, est de lui attribuer par sa conception et sa commande différents modes de déplacement. Ceux-ci lui permettent alors d'adapter le comportement de son système de propulsion de façon à optimiser les performances et le rendement énergétique de la tâche de locomotion.

L'idée d'utiliser des véhicules robotisés capables de se mouvoir suivant plusieurs modes de locomotion n'est apparue que récemment dans la littérature. Elle est souvent liée à la conception d'engins capables d'utiliser leurs organes de propulsion (roues, pattes, chenilles) suivant différentes combinaisons et à l'adaptation de ces modes en fonction du type d'obstacle à franchir. On peut citer, à titre d'exemple, les travaux sur le robot Hybtor [1], le Roller-walker [2] ou encore, plus récemment, le robot Azimut développé à l'Université de Sherbrooke [3]. 
Le choix et l'adaptation du mode de déplacement constituent la clé de voûte des systèmes de locomotion multi-modes, car les performances globales du véhicule pour l'exécution d'une mission donnée en dépendent. Ce choix suppose une comparaison à la fois qualitative et quantitative de ces différents modes de déplacement. Pour effectuer cette comparaison, il est nécessaire de définir des critères mesurant les performances de locomotion tels que la stabilité, et l'efficacité de la transmission de puissance à l'interface roue-sol. On s'intéresse donc ici à l'évaluation de ces critères pour la comparaison des modes de locomotion dans le cas particulier du robot Hylos.

Dans la deuxième section, nous présentons le robot hybride roue-patte Hylos et les modes de locomotion associés. Ensuite, la section suivante introduit les modèles du robot et de ces interactions avec le sol qui serviront à l'évaluation des différents modes de locomotion. Ces modèles d'interaction font l'objet de validations expérimentales présentées dans la quatrième section. Enfin, la cinquième section s'intéresse à l'évaluation, à partir des modèles analytiques, des performances de locomotion de chaque mode sur différents terrains.

\section{Description du robot Hylos et des modes de locomotion associés}

\subsection{Architecture mécatronique}

Le robot Hylos (Fig. 1) est un prototype expérimental de système de locomotion hybride roue-patte. Il est constitué d'une plate-forme à 4 roues-pattes possédant au total 16 degrés de liberté pilotés. Il mesure environ $70 \mathrm{~cm}$ de long pour un poids de $12 \mathrm{~kg}$. Chaque patte possède 2 liaisons pivot à axes parallèles, actionnées par des mécanismes à pantographe, et dispose à son extrémité d'une roue motrice et directrice. Il est équipé de 2 inclinomètres mesurant les angles de roulis et de tangage de la plate-forme, ainsi que de quatre capteurs d'effort 3 axes intégrés dans la structure de chaque patte et permettant la mesure des efforts d'interaction roue-sol. Le robot possède également des capteurs proprioceptifs (potentiomètres et codeurs optiques) à chaque articulation. Le système de commande du robot est distribué sur une architecture de calcul multi-processeurs permettant de distinguer les commandes bas-niveau de chaque patte, de la commande haut-niveau de l'ensemble du robot.

\subsection{Modes de locomotion}

La structure du mécanisme de locomotion des robots à propulsion hybride roue-patte permet d'envisager le déplacement du système suivant différents modes, en fonction de la combinaison de mouvements considérés entre les roues et les pattes. Il est possible d'imaginer un grand nombre de modes de déplacement. Cependant, en considérant la structure cinématique spécifique du robot Hylos, nous nous intéressons plus particulièrement dans cet article à trois modes qui peuvent être décrits suivant la nature du contexte opérationnel.

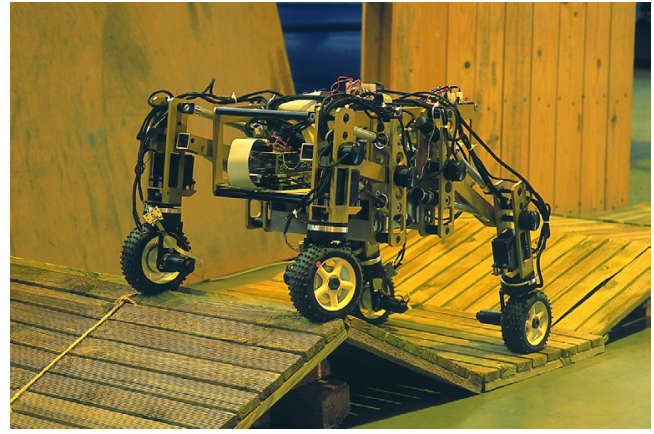

Fig. 1. Le robot Hylos.

\section{Mode 1}

Ce mode de « roulement simple» est un mode de déplacement trivial. Seules les roues sont utilisées dans ce mode et les mobilités internes restent fixes dans une configuration dite «nominale » illustrée sur la figure 2a. Il suppose que les mobilités internes puissent être verrouillées sans consommation d'énergie avec un système de blocage passif ou par l'utilisation de mécanismes de transmission irréversibles (de type transmission à vis, par exemple). Le mode par roulement simple sera employé pour déplacer le véhicule sur des sols relativement horizontaux ne présentant pas de fortes irrégularités ni de grandes discontinuités.

\section{Mode 2}

Dans le mode dit de « roulement avec reconfiguration », les mobilités internes sont utilisées afin de modifier la posture du robot lorsqu'il se déplace sur un terrain irrégulier. Une posture dite « sous-optimale » est déterminée en considérant l'optimisation des performances de locomotion. Elle vise principalement à atteindre une posture pour laquelle les composantes verticales des efforts de contact sont également distribuées sur les quatre roues [4]. La posture du robot est asservie au cours du déplacement sur cette posture sousoptimale en utilisant une loi de commande à retour d'état linéarisant [5]. Cette reconfiguration de posture permet, en outre, un ajustement de la garde au sol et une optimisation de la marge de stabilité.

\section{Mode 3}

C'est un mode séquentiel dit péristaltique. Il est inspiré par le mécanisme de locomotion de certains insectes comme la chenille [6]. La mise en œuvre de ce mode sur le robot Hylos consiste en une séquence de mouvements assurant le déplacement de chaque patte l'une après l'autre. Lors du déplacement des pattes, les mobilités de la patte et de la roue sont utilisées conjointement dans un schéma de commande en effort assurant une pression de contact constante et réglable. Le déplacement effectif du véhicule correspond au transfert du centre de masse du robot qui est obtenu par un actionnement coordonné des mobilités internes (les roues étant bloquées et fixes par rapport au 


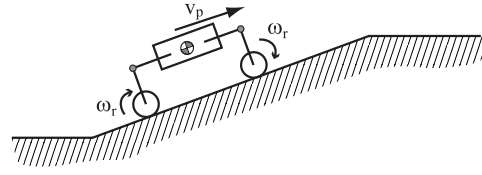

(a)

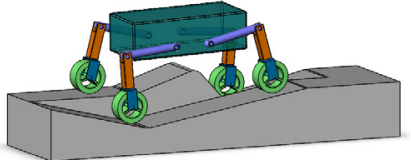

(b)

Fig. 2. Illustration des modes de déplacement par roulement. (a) Mode 1 : roulement simple. (b) Mode 2 : reconfiguration.

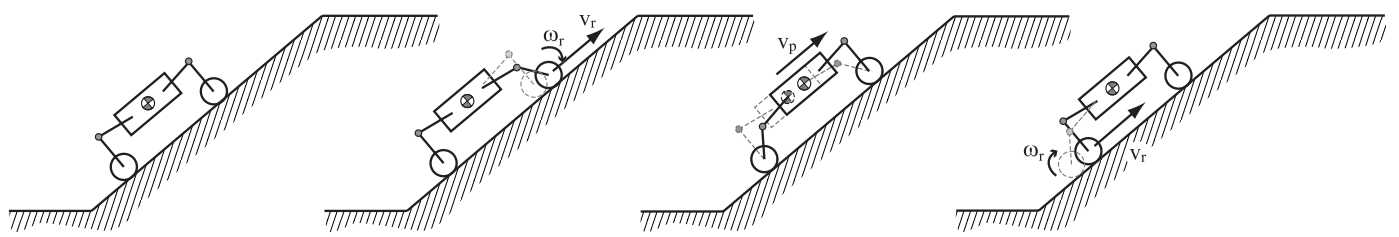

Fig. 3. Illustration du mode de déplacement séquentiel.

sol). La transmission de puissance dans la liaison roue-sol se fait donc lorsque les roues sont statiques, ce qui permet d'augmenter la capacité de propulsion même si l'efficacité de traction est faible pour le sol considéré. Ce mode de locomotion est donc plus particulièrement adapté au franchissement de pente dont le sol présente une faible cohésion (dune de sable, volcan, éboulis ...).

\section{Modélisation du système}

\subsection{Modèle cinémato-statique}

Nous nous intéressons ici à la modélisation du robot Hylos qui possède 4 chaînes roue-patte, chacune étant constituée de 4 liaisons rotoïdes actionnées. On note $\boldsymbol{x}^{\mathrm{t}}=\left(p^{\mathrm{t}}, \boldsymbol{\phi}^{\mathrm{t}}\right)$ le vecteur de dimension 6 , des paramètres opérationnels de la plate-forme où $\boldsymbol{p}=(x, y, z)^{\mathrm{t}}$ et $\phi=(\varphi, \psi, \theta)^{\mathrm{t}}$ qui sont, respectivement, la position $\mathrm{du}$ centre de gravité et l'orientation de la plate-forme. On note $\boldsymbol{q}=\left(\boldsymbol{q}_{1}, \ldots \boldsymbol{q}_{4}\right)^{\mathrm{t}}$ le vecteur, de dimension 16, des paramètres articulaires où $\boldsymbol{q}_{i}$ est le vecteur des paramètres du mécanisme de la $i^{\text {ème }}$ patte.

Le modèle cinématique du robot est obtenu en écrivant les conditions de glissement dans chaque liaison roue-sol. On note $\mathcal{R}_{i}=\left(P_{i}, \boldsymbol{t}_{i}, \boldsymbol{l}_{i}, \boldsymbol{n}_{i}\right)$ le repère de contact associé à la $i$ ème roue. Nous rappelons que $\boldsymbol{n}_{i}$ est le vecteur normal à la surface du terrain au point de contact $P_{i}$. Le vecteur $\boldsymbol{t}_{i}$ indique la direction longitudinale de la roue projetée dans le plan tangent du contact. Il est défini par l'intersection entre le plan médian de la roue et le plan tangent. Enfin, $\boldsymbol{l}_{i}=\boldsymbol{n}_{i} \times \boldsymbol{t}_{i}$ correspond à la direction latérale de la roue (Fig. 4b).

Les équations cinématiques qui relient les vitesses opérationnelles de la plate-forme $\dot{\boldsymbol{x}}$ aux vitesses articulaires $\dot{\boldsymbol{q}}_{i}$ de chaque chaîne roue-patte s'expriment sous la forme :

$$
\boldsymbol{L}_{i} \dot{\boldsymbol{x}}+\boldsymbol{J}_{i} \dot{\boldsymbol{q}}_{i}=\boldsymbol{v}_{\mathrm{g}_{i}}
$$

Dans cette équation, $\boldsymbol{L}_{i}$ et $\boldsymbol{J}_{i}$ sont respectivement la matrice de locomotion et la matrice jacobienne associée à chaque chaîne roue-patte et $\boldsymbol{v}_{\mathrm{g}_{i}}$ est le vecteur des vitesses de glissement de chaque roue. Le terme $\boldsymbol{L}_{i} \dot{\boldsymbol{x}}$ correspond à la vitesse du point de contact $P_{i}$ dans le mouvement de la plate-forme par rapport au sol et $\boldsymbol{J}_{i} \dot{\boldsymbol{q}}_{i}$ à la vitesse du point de contact dans le mouvement de la chaîne rouepatte par rapport à la plate-forme. Le modèle global s'exprime sous la forme :

$$
\boldsymbol{L} \dot{\boldsymbol{x}}+\boldsymbol{J} \dot{\boldsymbol{q}}=\boldsymbol{v}_{\mathrm{g}}
$$

De plus, les équations d'équilibres sont obtenues en appliquant le principe des travaux virtuels :

$$
\begin{aligned}
& \boldsymbol{L}^{\mathrm{t}} \boldsymbol{f}+\boldsymbol{w}=0 \\
& \boldsymbol{J}^{\mathrm{t}} \boldsymbol{f}+\boldsymbol{\tau}+\boldsymbol{w}_{\mathrm{s}}=0
\end{aligned}
$$

Dans ces équations, $\boldsymbol{f}=\left(\boldsymbol{f}_{i}\right)$ est le vecteur regroupant les composantes de forces de contact $\boldsymbol{f}_{i}$ exprimées dans le repère de contact de chaque roue $\mathcal{R}_{i}=\left(P_{i}, \boldsymbol{t}_{i}, \boldsymbol{l}_{i}, \boldsymbol{n}_{i}\right)$ et $\left(\boldsymbol{w}, \boldsymbol{w}_{\mathrm{s}}\right)$ sont les efforts généralisés dus au poids respectif de la plate-forme et de chaque sous-ensemble roue-patte [4].

\subsection{Modèle terra-mécanique}

Le modèle d'interaction développé dans cette partie définit les relations cinémato-statiques entre le déplacement relatif de la roue par rapport au sol et les efforts transmis dans cette liaison. On s'intéresse ici au cas particulier du robot Hylos, possédant des roues déformables et se déplaçant sur un sol meuble. Chaque roue étant motrice, on ne considère que le cas d'une roue soumise à un couple de traction. Nous présentons dans ce cadre spécifique, un modèle analytique explicite qui présente l'intérêt d'introduire les glissements et les efforts latéraux.

\section{Géométrie de contact}

La géométrie du contact définit l'aspect de la surface de contact entre la roue et le sol. Elle peut avoir une forme très complexe. Cependant, on considère généralement deux propriétés de forme pour définir la géométrie du contact : le profil de contact dans le plan médian de la 


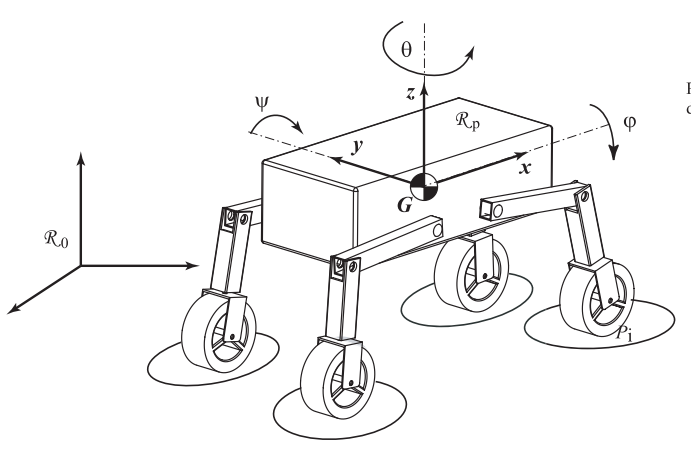

(a)

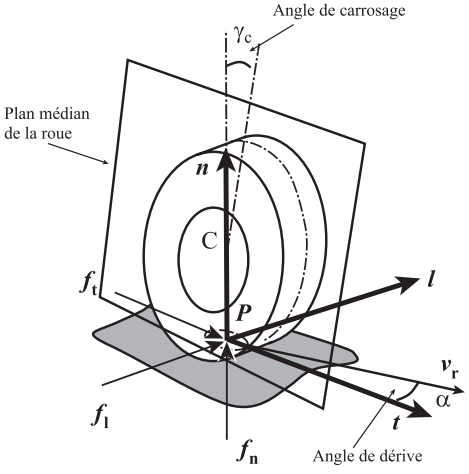

(b)

Fig. 4. Paramétrage du robot Hylos. (a) Géométrie du robot. (b) Repère de contact.

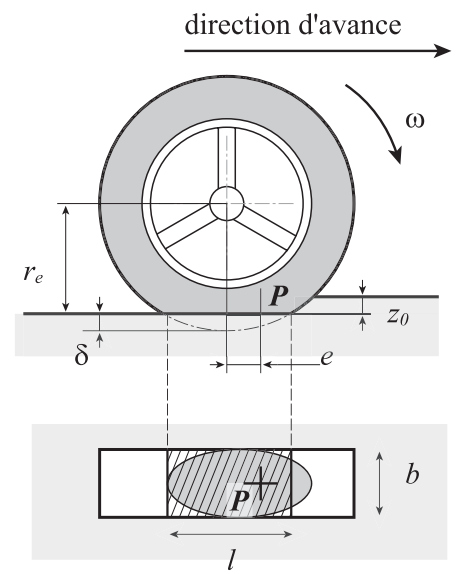

(a)

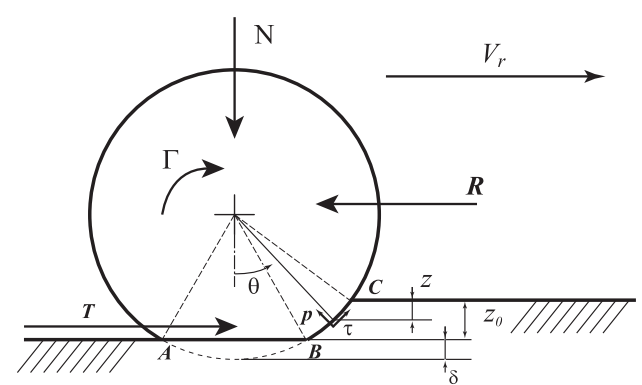

(b)

Fig. 5. Roue déformable sur sol meuble. (a) Géométrie du contact. (b) Efforts d'interaction.

roue, et la forme de l'empreinte de la roue dans le plan tangent du contact. Nous considérons dans ce modèle une empreinte rectangulaire de même largeur que la roue $b$ et de longueur $l$ [7]. De plus, le profil de contact est défini par un segment $[\mathrm{AB}]$ horizontal et un arc de cercle $[\mathrm{BC}]$ correspondant, respectivement, à la déformation de la roue et au tassement du sol (voir Fig. 5b).

\section{Efforts de contact}

Les efforts appliqués par le robot sur la roue sont la charge normale $N$ et le couple de traction $\Gamma$. Les efforts de contact $\boldsymbol{f}=\left(f_{\mathrm{t}}, f_{\mathrm{l}}, f_{\mathrm{n}}\right)^{\mathrm{t}}$ correspondant sont l'effort de poussée $f_{\mathrm{t}}=T-R$ générant le mouvement de la roue, la réaction normale du sol $f_{\mathrm{n}}=N$, l'effort latéral $f_{\mathrm{l}}=L$. $R$ est l'effort de résistance qui dépend de la nature physique du sol et de la caractéristique en déformation de la roue. $T=\Gamma / r_{\mathrm{e}}$ est l'effort de traction appliqué par la roue sur le sol. De plus, on considère que les moments propres dans la liaison roue-sol sont négligeables.

Les efforts transmis dans la liaison roue-sol correspondent à l'intégration des contraintes le long de l'interface de contact (l'empreinte de contact étant rectangulaire, la répartition des efforts ne dépend pas de $y$ ).
L'équilibre des forces du système en projection suivant les vecteurs de base du repère de contact $\mathcal{R}_{i}$ conduit à :

$$
\begin{aligned}
& \left.N=b\left(\int_{A}^{C} r p(\theta) \cos \theta \mathrm{d} \theta+\int_{A}^{C} r \tau(\theta) \sin \theta \mathrm{d} \theta\right)\right) \\
& T=b \int_{A}^{C} r \tau(\theta) \cos \theta \mathrm{d} \theta \\
& R=b \int_{A}^{C} r p(\theta) \sin \theta \mathrm{d} \theta
\end{aligned}
$$

Dans ces équations, $p$ est la contrainte de pression qui varie en fonction de l'enfoncement dans le sol $z$ suivant l'équation de Bekker [8] :

$$
p(z)=\left(k_{\mathrm{c}} / b+k_{\phi}\right) z^{n}=k_{\mathrm{eq}} z^{n}
$$

et $\tau$ est la contrainte de cisaillement qui dépend du déplacement de cisaillement $j$ suivant le modèle de Janosi [9] :

$$
\tau(j)=\tau_{\max }\left[1-\exp \left(-\frac{j}{K}\right)\right]
$$

où $k_{\text {eq }}$ est la raideur équivalente du sol, $n$ l'exposant d'enfoncement et $K$ le module de cisaillement. 
Sous l'action de l'effort normal de contact $N$ et du couple de traction $\Gamma$, la roue et le sol subissent une déformation. Dans le plan vertical, il s'agit de la relation entre l'enfoncement combiné à l'écrasement de la roue et la charge normale $N$. Dans le plan tangent, il s'agit des relations entre effort de cisaillement et taux de glissement.

\section{Enfoncement et écrasement de la roue}

La relation (5) se décompose en la somme de deux fonctions intégrales : $N=N_{\mathrm{AB}}+N_{\mathrm{BC}}$. Dans la section $[\mathrm{AB}]$, le sol est soumis à une pression constante $p_{\mathrm{r}}$ qui est la somme des pressions dues à la pression du pneumatique et de la carcasse du pneu, telle que $N_{\mathrm{AB}}=b l p_{\mathrm{r}}$. La déformation de la roue $\delta$ en fonction de la charge normale suit une loi empirique quasi-linéaire du type : $\delta=k_{\mathrm{r}} N$. Par ailleurs, la pression $p_{\mathrm{r}}$ est égale à la contrainte verticale subie par le sol et peut être exprimée en fonction de la compaction du terrain $z_{0}$, en utilisant le modèle de Bekker (8) :

$$
z_{0}=\left(\frac{p_{\mathrm{r}}}{k_{\mathrm{c}} / b+k_{\phi}}\right)^{1 / n}=\left(\frac{p_{\mathrm{r}}}{k_{\mathrm{eq}}}\right)^{1 / n}
$$

Dans la partie circulaire $[\mathrm{BC}]$ les expressions de contraintes peuvent s'écrire sous la forme :

$$
\left\{\begin{array}{l}
r p \cos \theta \mathrm{d} \theta=p \mathrm{~d} x \\
r \tau \sin \theta \mathrm{d} \theta=\tau \mathrm{d} z
\end{array}\right.
$$

La pression $p$ s'exprime en fonction de la profondeur d'enfoncement dans le sol suivant la loi de comportement de l'équation de Bekker, et on suppose que la contribution $\tau \mathrm{d} z$ est négligeable :

$$
N_{\mathrm{BC}}=b \int_{0}^{z_{0}}\left(k_{\mathrm{eq}}\right) z^{n} \mathrm{~d} x
$$

L'intégration de cette fonction à l'aide d'un développement en série au second ordre donne la relation entre l'effort normal $N$ et l'enfoncement combiné (dû au tassement du sol $z_{0}$ et à la déformation $\delta$ de la roue) [10] :

$$
\begin{array}{r}
N=k_{\mathrm{r}} \delta+\frac{b}{3} k_{\mathrm{eq}} \sqrt{D}\left(z_{0}+\delta\right)^{n-1}\left[(3-n)\left(z_{0}+\delta\right)^{3 / 2}\right. \\
\left.-(3-n) \delta^{3 / 2}-3 z_{0} \sqrt{\delta}\right]
\end{array}
$$

\section{Résistance au roulement}

La résistance au roulement correspond à la perte d'énergie due à la compaction du sol lorsque la roue est en mouvement. Elle dépend de la profondeur d'enfoncement du sol et donc de la pression de contact :

$$
R=b \int_{\mathrm{B}}^{\mathrm{C}} p r \sin \theta \mathrm{d} \theta=b \int_{0}^{z_{0}} p \mathrm{~d} z=b \frac{k_{\mathrm{eq}}}{n+1} z_{0}^{n+1}
$$

La résistance au roulement s'exprime donc en fonction de la pression de contact $p_{\mathrm{r}}$ sous la forme :

$$
R=b \frac{p_{\mathrm{r}}^{(n+1) / n}}{(n+1) k_{\mathrm{eq}}^{1 / n}}
$$
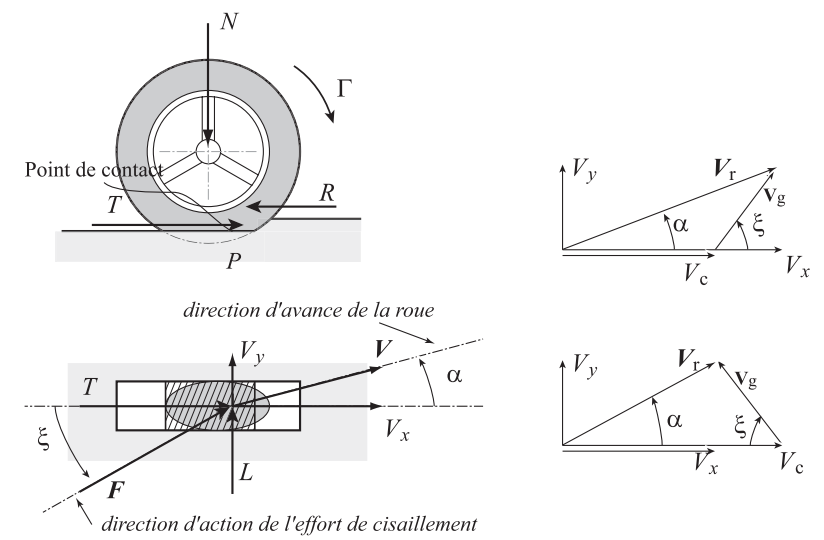

Fig. 6. Relations entre efforts de cisaillement et vitesse de glissement.

\section{Vitesse de glissement}

Soit $\boldsymbol{v}_{\mathrm{r}}$, la vitesse de déplacement de la roue par rapport au sol. Elle est orientée d'un angle $\alpha$ par rapport à la direction longitudinale $\boldsymbol{t}$ de la roue. $\alpha$ est l'angle de dérive de la roue. On note $V_{x}$ la composante de la vitesse $\boldsymbol{v}_{\mathrm{r}}$ projetée suivant l'axe longitudinal $\boldsymbol{t}$ et $V_{y}$ sa composante suivant l'axe latéral $\boldsymbol{l}$ (Fig. 6).

La vitesse de glissement $\boldsymbol{v}_{\mathrm{g}}$ est la différence entre la vitesse de déplacement de la roue $\boldsymbol{v}_{\mathrm{r}}$ par rapport au sol et sa vitesse de circonférence $\boldsymbol{v}_{\mathrm{c}}=V_{\mathrm{c}} \boldsymbol{t}$. Le glissement de la roue par rapport au sol est généralement caractérisé par le taux de glissement longitudinal $s$ et l'angle de dérive $\alpha$ :

$$
s= \begin{cases}\frac{V_{\mathrm{c}}-V_{x}}{V_{c}}>0 & \text { en traction } \\ \frac{V_{\mathrm{c}}-V_{x}}{V_{x}}<0 & \text { en freinage }\end{cases}
$$

\section{Effort de cisaillement}

La plupart des modèles d'interaction tels que le modèle de Bekker ne prennent pas en compte le glissement latéral et l'angle de dérive. Dans le modèle de Bekker, l'effort de traction est obtenu en intégrant les contraintes de cisaillement exprimées sur la base du modèle de Janosi (9), soit :

$$
T=\int_{\text {A }}^{\mathrm{C}} r \tau(\theta) \mathrm{d} \theta=\int_{\mathrm{A}}^{\mathrm{C}} \tau(x) \mathrm{d} x
$$

En rappelant que l'expression du taux de glissement est $s=\mathrm{d} j / \mathrm{d} x$, on obtient :

$$
T=T_{\max }\left(1-\frac{K}{s l}\left[1-\exp \left(\frac{-s l}{K}\right)\right]\right)
$$

En tenant compte de l'angle de dérive, l'effort de cisaillement $\boldsymbol{F}$ est supposé colinéaire avec la vitesse de glissement $\boldsymbol{v}_{\mathrm{g}}$ :

$$
\left\{\begin{array}{l}
T=F \cos \xi \\
L=F \sin \xi
\end{array}\right.
$$


On montre alors que les composantes d'effort dans le plan tangent ( $T$ et $L$ ) s'expriment en fonction des paramètres de glissement $s$ et $\alpha$ qui sont respectivement le taux de glissement longitudinal et l'angle de dérive [10] :

$$
\left\{\begin{array}{l}
\tan \xi=\frac{1-s}{s} \tan \alpha \\
u=\sqrt{(1-s)^{2} \tan ^{2} \alpha+s^{2}} \\
F=(A c+N \tan \phi)\left[1-\frac{K}{u l}\left(1-\exp \left(\frac{-u l}{K}\right)\right)\right]
\end{array}\right.
$$

où $u$ est le taux de glissement suivant la direction de $\boldsymbol{v}_{\mathrm{g}}$, $c$ exprime la cohésion du sol et $\phi$ son angle de frottement, et $A$ est l'aire de contact $[7,10]$.

\section{Validation expérimentale du modèle d'interaction roue-sol}

\subsection{Dispositif expérimental}

Nous avons mis en œuvre une procédure de caractérisation du modèle d'interaction roue-sol sur un banc expérimental, développé spécifiquement pour ce problème (Fig. 7). Il permet de réaliser des expériences en laboratoire pour une identification préalable des paramètres d'interaction pour un couple roue-sol donné. Nous avons ainsi analysé le comportement des roues du robot Hylos en interaction avec un sol de type sable sec.

Ce dispositif expérimental est composé d'un chariot, animé d'un mouvement horizontal et piloté par un motoréducteur à courant continu, sur lequel est fixée une glissière verticale non-actionnée. Une roue motorisée est fixée à l'extrémité de la glissière par l'intermédiaire d'un capteur d'effort 6 axes. La roue est pilotée en traction par un moto-réducteur. Il est possible de modifier l'orientation de l'ensemble composé de la roue tractive et du capteur d'effort par une rotation d'axe vertical. Ceci permet de simuler un angle de dérive $\alpha$ entre la direction d'avance de la roue et son axe longitudinal. Le capteur d'effort permet de mesurer les 6 composantes de l'action mécanique entre le support roue et la glissière, et donc d'estimer les composantes d'action mécanique à l'interaction roue-sol. Compte tenu de la nature du montage, les composantes des efforts sont mesurées directement dans un repère associé à la roue.

Le chariot horizontal est asservi en vitesse et permet ainsi d'imposer une vitesse d'avance constante à la roue. De même, la vitesse de rotation de la roue est asservie. Il est donc possible d'imposer les conditions cinématiques de glissement du couple roue-sol, à savoir le glissement longitudinal $s$ et l'angle de dérive $\alpha$. Le glissement longitudinal est fixé en imposant une différence entre la vitesse du chariot et le taux de rotation de la roue, on peut ainsi simuler les phases de freinage et d'accélération avec différents taux de glissement. La figure 8 donne une représentation schématique du dispositif expérimental.
La glissière verticale est équipée d'un capteur de déplacement linéaire de type résistif permettant la mesure de la position verticale de la roue et donc de l'enfoncement de celle-ci dans le sol.

Ce dispositif permet donc de faire varier les conditions de l'interaction roue-sol en terme de déplacement :

- vitesse d'avance $V_{\mathrm{r}}$,

- vitesse de rotation de la roue $\omega$ (et donc le glissement longitudinal $s$ ),

- angle de dérive $\alpha$,

et permet de mesurer les paramètres suivants :

- les efforts de contact $\left(f_{\mathrm{t}}, f_{\mathrm{l}}, f_{\mathrm{n}}\right)$,

- l'écart de position vertical de la roue $z_{t}=z_{0}+\delta$ (donc de l'enfoncement combiné de la roue et du sol).

\section{2 Étude du comportement en compression}

Nous avons utilisé le banc d'essai afin de caractériser le comportement spécifique de l'interaction des roues du robot Hylos sur du sable sec.

\section{Caractéristique en déformation de la roue seule}

Comme nous l'avons indiqué précédemment, une condition préalable à la caractérisation du comportement roue-sol, est l'identification de la loi de déformation de la roue. Pour cela, nous procédons à une expérience à l'aide du banc d'essai : la roue est placée sur une surface rigide, et l'expérience consiste à mesurer la variation de l'effort normal en fonction de l'écrasement de la roue (mesuré avec le capteur de position du chariot vertical). Nous obtenons alors la relation $f_{\mathrm{n}}=f(\delta)$ représentée sur le graphe de la figure 9 qui s'identifie à une droite $f_{\mathrm{n}}=k_{\mathrm{r}} \delta$. Dans le cas des roues utilisées sur le robot Hylos, on obtient $k_{\mathrm{r}} \approx 6 \mathrm{kN} \cdot \mathrm{m}^{-1}$.

\section{Comportement en compression du sol}

Pour la caractérisation du comportement vertical du sol, nous utilisons le même dispositif que précédemment en remplaçant la roue par un plateau rigide. Les dimensions $(40 \times 60 \mathrm{~mm})$ sont proches de celles de la roue. Ainsi, nous pouvons caractériser les propriétés de raideur propres au sol. Nous rappelons que la loi de comportement vertical du sol est de la forme $p=\left(k_{\mathrm{c}} / b+k_{\phi}\right) z^{n}=$ $k_{\mathrm{eq}} z^{n}$. Dans cette expérience, $b=40 \mathrm{~mm}$ est la plus petite dimension du plateau et elle est égale à la largeur de la roue. Ainsi, la raideur équivalente du sol $k_{\text {eq }}=\left(k_{\mathrm{c}} / b+k_{\phi}\right)$ déterminée dans cette expérience sera aussi valable pour les roues du robot. Il n'est donc pas nécessaire d'évaluer indépendamment les paramètres $k_{\mathrm{c}}$ et $k_{\phi}$. Notons cependant qu'il suffit de faire une série de mesure avec des plateaux de tailles différentes afin d'extraire les deux paramètres.

Le graphe de la figure 10 représente la relation entre l'effort normal $f_{\mathrm{n}}$ et le déplacement vertical $z_{0}$ du plateau mesurés lors de l'expérience. La surface de contact $A$ est constante, on obtient une relation de la forme $f_{\mathrm{n}}=$ $A k_{\mathrm{eq}} z^{n}$ avec $n=1$ et $k_{\mathrm{eq}}=1,54 \times 10^{3} \mathrm{kN} . \mathrm{m}^{-3}$. 


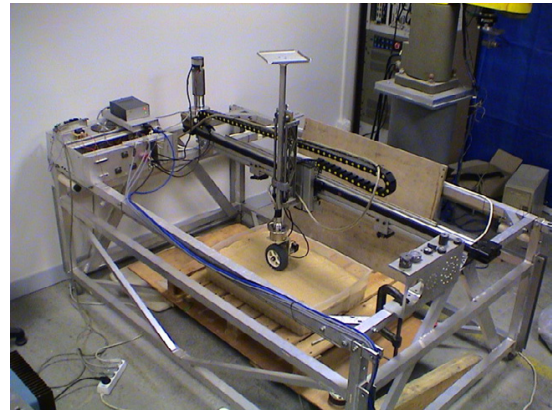

(a)

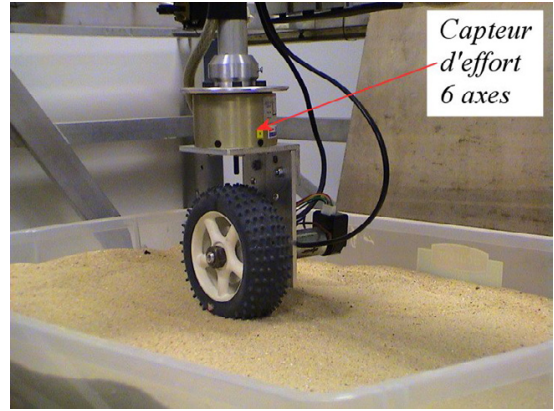

(b)

Fig. 7. Banc d'essai pour la caractérisation des interactions roue-sol. (a) Vue d'ensemble. (b) Roue de traction avec capteur d'efforts.

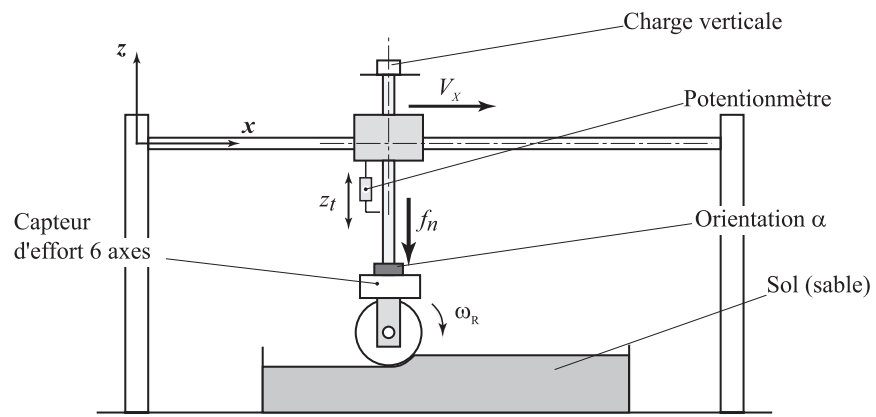

Fig. 8. Vue schématique du dispositif expérimental.

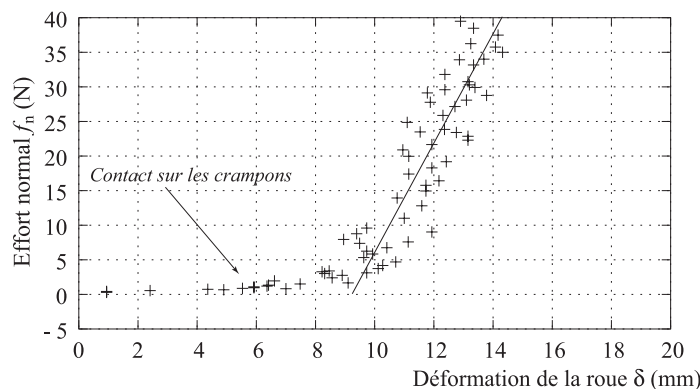

Fig. 9. Caractérisation du comportement de la roue flexible.

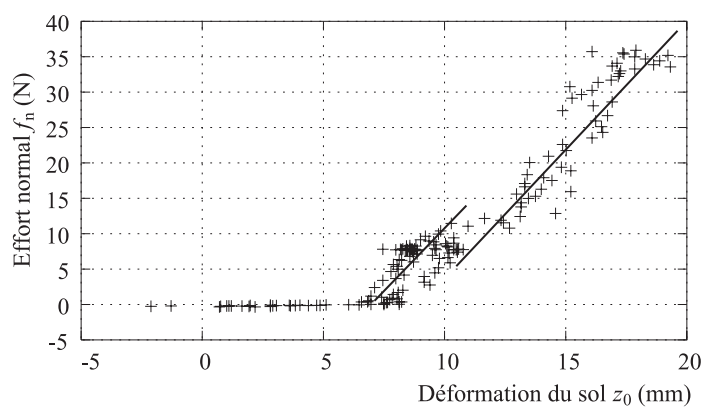

Fig. 10. Enfoncement d'un plateau rigide dans le sol.

\section{Caractérisation du système roue-sol}

Dans cette expérience, le déplacement vertical de la roue est imposé et l'effort vertical est mesuré par le capteur d'effort. Cet effort engendre un écrasement de la roue combiné à la compaction du sol $z_{\mathrm{t}}=z_{0}+\delta$.

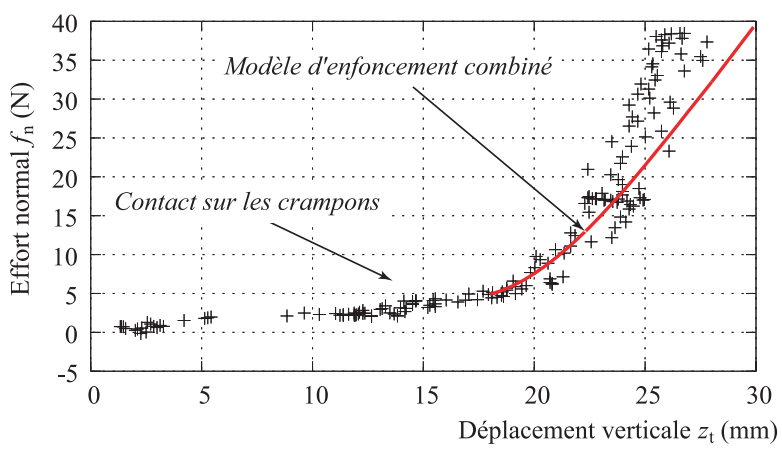

Fig. 11. Enfoncement d'une roue flexible sur du sable.

Le comportement du système roue-sol est caractérisé par le modèle présenté dans l'équation (11) qui avec $n=1$ devient :

$$
f_{\mathrm{n}}=k_{\mathrm{r}} \delta+b k_{\mathrm{eq}} \sqrt{D}\left(\frac{2}{3} z_{\mathrm{t}}^{3 / 2}-\left(z_{\mathrm{t}} \sqrt{\delta}-\frac{1}{3} \delta^{3 / 2}\right)\right)
$$

L'objet de cette expérience est de valider le modèle proposé. Pour cela, on compare les efforts mesurés en fonction de l'enfoncement $z_{\mathrm{t}}$ par rapport à ceux estimés par le modèle. La courbe de la figure 11 présente les résultats obtenus. La zone d'amorce de la courbe montre une raideur très faible qui correspond à la phase de pénétration des crampons dans le sable. On vérifie que le modèle proposé à la section 3.2 est représentatif du comportement observé dans la plage d'enfoncement $z_{\mathrm{t}} \in[17,25] \mathrm{mm}$. Au-delà de cette valeur, l'allure de la courbe change pour devenir «plus » linéaire. En effet, à partir d'une certaine valeur d'écrasement $\delta$, la roue ne se déforme plus. La courbe d'enfoncement devient linéaire et correspond au tassement du sol par la jante qui est rigide par rapport à celui-ci.

\section{3 Étude du comportement en cisaillement}

\section{Caractéristique du sol}

Nous nous intéressons, maintenant, aux propriétés de cisaillement de l'interaction roue-sol. La première 


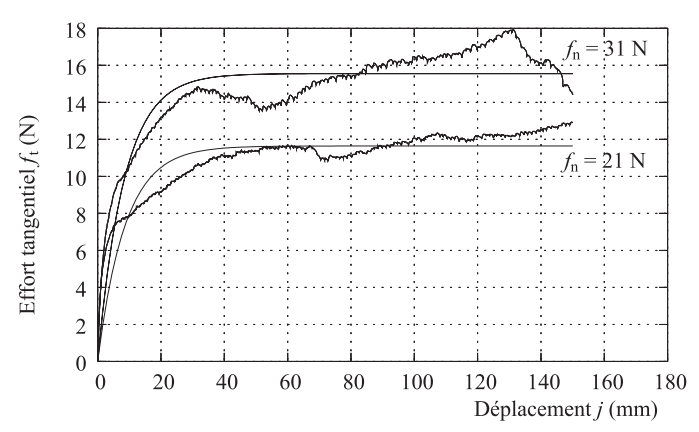

Fig. 12. Comportement d'un sol de type sable en réponse à un déplacement horizontal.

expérience que nous avons réalisée consiste en un test de cisaillement du sol. Pour cela, nous utilisons une roue rigide à laquelle on applique une rotation à très faible vitesse et le chariot horizontal reste immobile. Ainsi, la rotation de la roue entraîne un déplacement relatif du point de contact $P$ appartenant à la roue par rapport au sol. Il est alors possible de calculer le déplacement $j$ au niveau du point de contact :

$$
j(t)=\int_{0}^{t} R \omega \mathrm{d} t
$$

L'effort vertical constant est imposé par une masse disposée sur la glissière verticale. L'effort tangentiel et l'effort normal sont mesurés par le capteur d'effort et on obtient les courbes de la figure 12. L'effort tangentiel correspond à la contrainte de cisaillement et on retrouve la loi de comportement en exponentielle proposé par Janosi (Éq. (9)).

Cette expérience nous permet d'identifier le module de cisaillement $K$, l'angle de frottement $\phi$ et le paramètre de cohésion $c$. Le module $K$ correspond à la pente à l'origine de la courbe de cisaillement et les paramètres de cohésion $c$ et de frottement $\phi$ sont obtenus en évaluant l'effort tangentiel $T_{\max }$ pour différentes valeurs de la charge normale $N$.

\section{Système roue-sol}

L'objet de cette expérience est de caractériser directement la relation entre les efforts de cisaillement et les paramètres de glissement à l'interface roue-sol. Nous considérons ici uniquement le cas où la vitesse relative de la roue par rapport au sol ne présente pas de dérive $\alpha=0$. Le taux de glissement $s$ est imposé par la différence entre les vitesses appliquées à la roue $V_{\mathrm{c}}=r \omega$ et au chariot $V_{\mathrm{r}}$. On effectue des séries de mesure à glissement constant et à charge normale constante. Entre chaque série, on fait varier le glissement. Le résultat de ces mesures est illustré par les courbes de la figure 13. Par la méthode des moindres carrés, le modèle d'interaction de l'équation (13) permet d'identifier le module de cisaillement $K$ du couple roue-sol, la résistance au roulement $R$, et l'effort de cisaillement maximal $T_{\max }$ :

$$
f_{\mathrm{t}}=T-R=T_{\max }\left(1-\frac{K}{s l}\left[1-\exp \left(\frac{-s l}{K}\right)\right]\right)-R
$$

L'évaluation de $T_{\max }$ en fonction de $f_{\mathrm{n}}$ permet d'obtenir les paramètres $c$ et $\phi$ :

$$
T_{\max }=A c+f_{\mathrm{n}} \tan \phi
$$

\section{5 Évaluation des performances de locomotion}

Dans cette section, nous proposons de qualifier la mobilité du robot Hylos en fonction des paramètres du sol. Nous rappelons que l'objectif est d'adapter les modes de déplacement en fonction des conditions opérationnelles du robot. Dans cette étude, les paramètres de sol sont supposés connus et on considère à titre d'exemple les deux sols décrits dans le tableau 1. Les modèles cinémato-statique et terra-mécanique sont utilisés pour évaluer les performances de déplacement du robot. Les résultats obtenus sont propres au robot Hylos, puisque certains paramètres structuraux (dimensions du robot) et opérationnels (puissance des actionneurs) ont un impact sur les critères de performance. Néanmoins, ces résultats nous permettent de dégager quelques règles générales, d'un point de vue qualitatif, sur l'adéquation entre les modes de déplacement et le type de terrain.

\subsection{Marge de stabilité}

Pour l'étude de la stabilité du robot, nous utilisons une marge de type gradient [11]. Le principe consiste à calculer les angles de stabilité $v_{i}$ qui définissent la marge de stabilité du robot autour de chacun de ces axes de renversement $\hat{\boldsymbol{a}}_{i}$ reliant deux points de contact $P_{i}$ consécutifs. Pour cela, on détermine la contribution au basculement $\boldsymbol{f}_{i}^{*}$ du torseur $\boldsymbol{w}_{t}$ des efforts appliqués au robot, autour de chacun de ces axes. On note $M_{\mathrm{s}}$ la marge de stabilité globale du véhicule qui est définie comme étant la valeur minimale de l'ensemble des $v_{i}$ avec $i=\{1, \ldots, n\}$ :

$$
M_{\mathrm{S}}=\min \left(v_{i}\right) \quad \text { pour } \quad i=\{1, \ldots, n\}
$$

Cette grandeur scalaire constitue donc une mesure instantanée de la marge de stabilité du véhicule. Lorsque la métrique $M_{\mathrm{s}}$ est positive le système est d'autant plus stable que la valeur de $M_{\mathrm{s}}$ est grande. Si $M_{\mathrm{s}}$ est nulle le système est en limite de basculement. L'optimisation de la marge de stabilité consiste à rendre cette mesure maximale.

L'objet de ce paragraphe est de montrer, à l'aide d'une métrique caractérisant la marge de stabilité mécanique du robot, l'apport de la reconfiguration dans les modes de locomotion par roulement. Pour cela, nous calculons la marge de stabilité du robot en position sur un terrain incliné. Le calcul de la marge de stabilité est réalisé pour différents angles $\theta$ d'orientation du robot par rapport à l'axe de plus forte pente. L'idée est d'évaluer la stabilité à la fois sur des pentes frontales $(\theta=0)$ et sur des pentes en dévers $(\theta= \pm \pi / 2)$, ainsi que pour toutes les configurations intermédiaires. Notons que pour cette analyse la nature physique du sol n'a pas d'influence. 

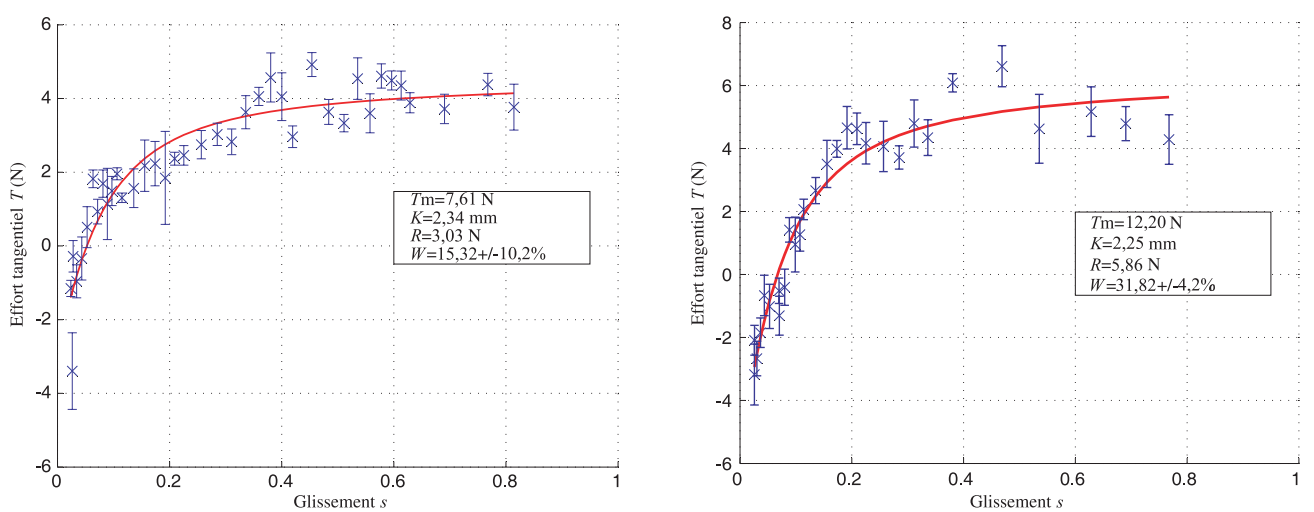

Fig. 13. Caractérisation de la relation traction-glissement pour une roue flexible.

Tableau 1. Paramètres de sol utilisés pour l'évaluation.

\begin{tabular}{llcccccc}
\hline Id & Terrain & $n$ & $K_{\mathrm{c}}\left(\mathrm{kN} / \mathrm{m}^{n+1}\right)$ & $K_{\phi}\left(\mathrm{kN} / \mathrm{m}^{n+2}\right)$ & $c(\mathrm{kPa})$ & $\Phi(\mathrm{deg})$ & $K(\mathrm{~cm})$ \\
\hline 1 & Sable sec & 1,10 & 0,99 & 1528,43 & 1,04 & 28,0 & 0,70 \\
2 & Sable compact & 0,79 & 102,0 & 5301,00 & 1,30 & 31,1 & 1,14 \\
\hline
\end{tabular}

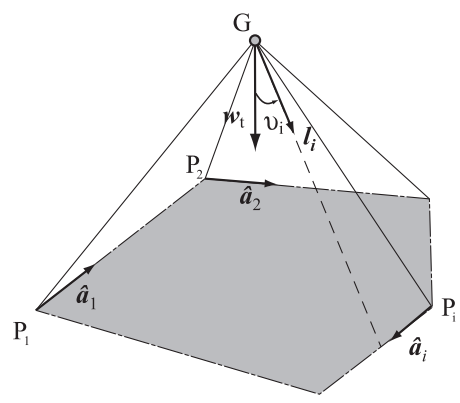

Fig. 14. Marge de stabilité de type gradient.

La figure 15 représente la limite de stabilité sur un graphique polaire où le rayon correspond à l'angle de pente $\eta$ et l'angle polaire est l'angle d'orientation $\theta$ par rapport à la pente. On constate que le mode 2 offre une limite de stabilité bien supérieure aux autres modes, en particulier quand le robot évolue sur une pente en dévers. En effet, l'apport de la reconfiguration est plus sensible en devers que sur une pente frontale car les dimensions du robot sont anisotropes (le robot est plus long que large). Le comportement irrégulier des courbes en modes 1 et 3 s'explique aussi par cette différence de dimension du robot. Par contre dans le mode 2, le comportement irrégulier est principalement dû aux limites de l'espace de travail des pattes.

\subsection{Gradabilité}

La gradabilité s'intéresse à la capacité de franchissement d'un sol plan incliné. Le comportement du véhicule se déplaçant sur ce type de terrain dépend de la configuration du système véhicule-pente. Elle est définie par deux paramètres qui sont l'angle de pente $\eta$ et l'angle d'orientation de la direction longitudinale du robot par rapport à la direction de plus forte de pente $\theta_{\mathrm{p}}$ (Fig. 15b).
Pour une orientation donnée $\theta$ du robot par rapport à l'axe de plus grande pente, il est possible de calculer à l'aide du modèle quasi-statique les efforts au contact $\boldsymbol{f}_{i}=$ $\left(f_{\mathrm{t}}, f_{\mathrm{l}}, f_{\mathrm{n}}\right)^{\mathrm{t}}$ sur chaque roue. Ainsi, pour un terrain donné, le calcul des efforts de contact nous permet d'évaluer les paramètres de glissement de chaque roue.

L'effort normal nous donne la géométrie de contact (écrasement de la roue $\delta$ et compaction du sol $z_{0}$ ) et la résistance au roulement $R$ est donnée par l'équation (12). Ceci permet alors de calculer les efforts de cisaillement $T$ et $L$. Les paramètres de glissement sont fonctions de l'effort de cisaillement total $F=\sqrt{T^{2}+L^{2}}$ et de son angle de direction $\xi=\arctan (T / L)$. Le taux de glissement $u$ dépend de l'effort de cisaillement suivant l'équation (15) qui ne présente pas de solution analytique. Cependant, il est possible de calculer, avec une bonne approximation, le taux de glissement $u$ en fonction de $F$, en utilisant une formulation logarithmique telle que celle introduite dans [12] :

$$
u=\frac{-K / l}{\ln \left(F / F_{\max }\right)}
$$

L'angle de dérive $\alpha$ s'obtient avec l'équation (15). On obtient alors les paramètres de glissement pour chaque configuration de pente $(\eta, \theta)$.

$$
\begin{aligned}
& s=u \cos \xi \\
& \tan \alpha=\frac{s}{1-s} \tan \xi=\frac{s}{1-s} \frac{L}{T}
\end{aligned}
$$

La gradabilité est caractérisée par la pente limite que le robot peut franchir, en maintenant un niveau suffisant de maîtrise des mouvements du robot (i.e. la contrôlabilité). La contrôlabilité est définie en considérant, à la fois, le taux de glissement et l'angle de dérive admissibles sur chaque roue; ils sont notés respectivement $s^{*}$ et $\alpha^{*}$. Le critère de gradabilité correspond au plus faible de ces deux angles de pente limite :

$$
C_{\mathrm{g}}=\min \left(\eta_{s}^{*}, \eta_{\alpha}^{*}\right)
$$




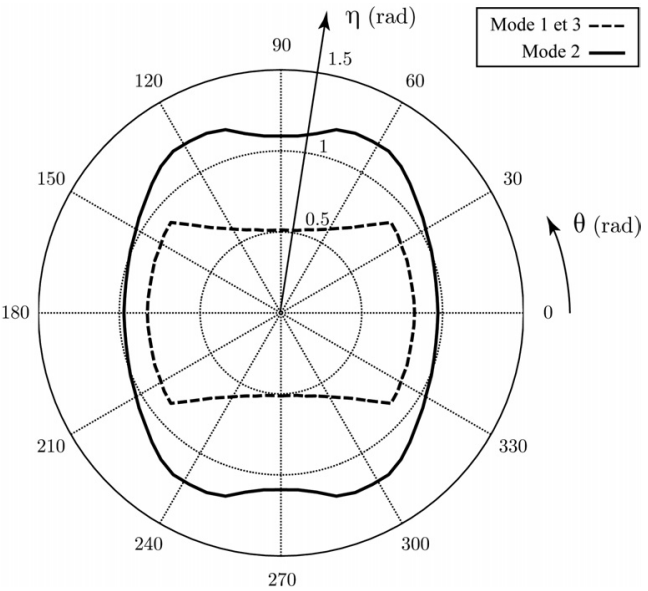

(a)

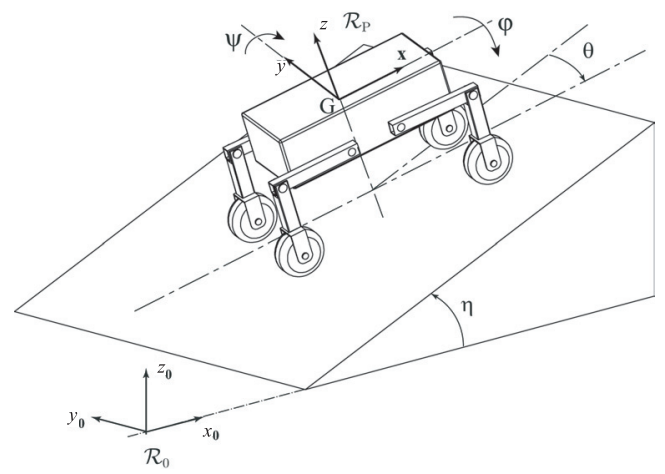

(b)

Fig. 15. Limite de stabilité pour différentes configurations de pente.

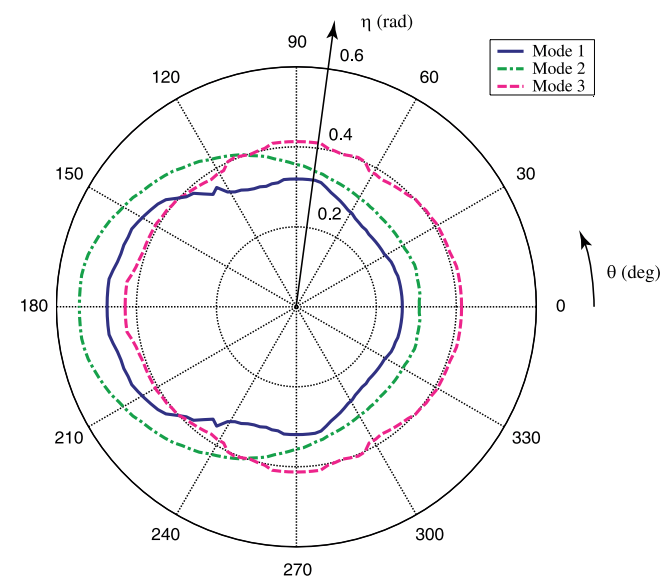

(a)

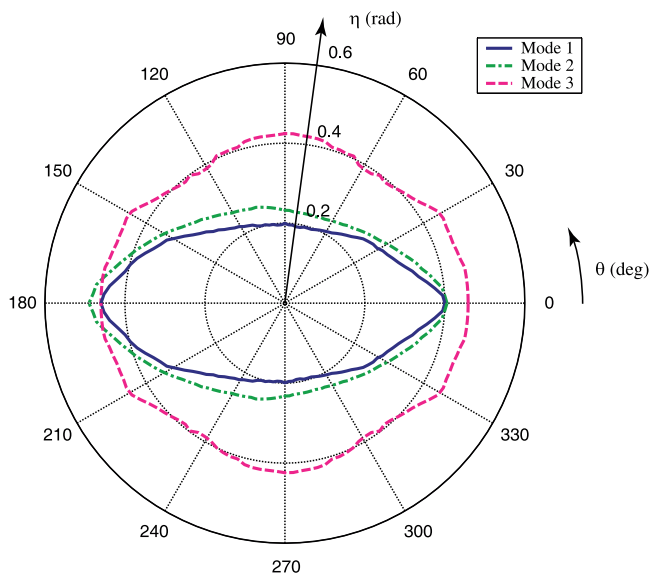

(b)

Fig. 16. Courbes de gradabilité du robot. (a) Terrain type 1 (sable sec). (b) Terrain type 2 (sable compact).

La figure 16 représente sur un graphique polaire (comme pour l'étude de la marge de stabilité) les limites de gradabilité pour les sols de types 1 et 2 dont les paramètres sont donnés dans le tableau 1 . Dans cette étude, on fixe également des valeurs limites de 0,5 pour le taux de glissement $s$ et de $15^{\circ}$ pour l'angle de dérive $\alpha$.

Nous observons que le mode péristaltique offre de bonnes capacités de franchissement sur des pentes fortement inclinées. L'avantage principal de ce mode est qu'il réduit au minimum la traction par roulement et donc la résistance au roulement qui est due au tassement du sol. De plus, dans ce mode et au contraire des autres modes, la gradabilité ne dépend pas de l'angle d'orientation du robot. En modes 1 et 2, les efforts latéraux présents pour les configurations de pente en dévers, affectent fortement les performances en terme de gradabilité du système. En outre, les courbes ne sont pas symétriques par rapport à l'axe vertical $\left(\theta= \pm 90^{\circ}\right)$ car, lorsque le véhicule descend sur une pente, la résistance de roulement contribue cette fois à améliorer la gradabilité.

\subsection{Consommation d'énergie}

On considère, avec ce critère, la consommation d'énergie du robot lors de son déplacement sur un terrain donné. Celle-ci est définie comme étant la somme des contributions énergétiques, de chaque actionneur, à la mise en mouvement du robot (articulations internes et traction des roues). La puissance consommée par un actionneur $P_{i}$ dépend de sa vitesse $\omega_{i}$ et du couple $\Gamma_{i}$ qu'il génère $P_{i}(t)=\omega_{i}(t) \Gamma_{i}(t)$. En intégrant la puissance fournie par l'actionneur le long de la trajectoire, de l'instant initial $t_{0}=0$ jusqu'à l'instant final $t_{1}=t_{\mathrm{f}}$, on obtient l'énergie totale consommée par le robot :

$$
E_{\mathrm{t}}=\sum_{i} E_{i}=\sum_{i}\left(\int_{0}^{t_{\mathrm{f}}} \omega(t) \Gamma_{i}(t) \mathrm{d} t\right)
$$

Le critère utilisé pour l'évaluation des modes de propulsion est la consommation d'énergie par unité de distance parcourue :

$$
C_{\mathrm{p}}=\frac{E_{\mathrm{t}}}{L}
$$




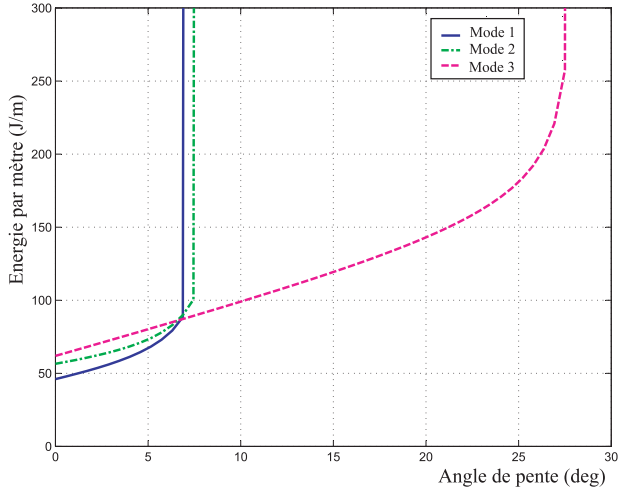

(a)

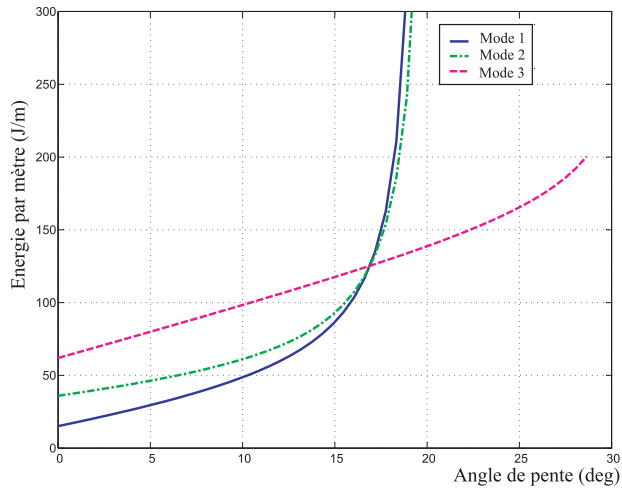

(b)

Fig. 17. Consommation d'énergie en fonction de la pente. (a) Terrain de type 1. (b) Terrain de type 2.

Nous analysons ce critère d'énergie lors du déplacement du robot sur une pente frontale $\left(\theta=0^{\circ}\right)$ pour chaque mode et pour chaque terrain du tableau 1 . Les courbes de la figure 17 représentent l'énergie par unité de distance parcourue en fonction de l'angle de pente $\eta$. Nous pouvons noter que le mode 1 est le plus efficace pour des angles de pente faible, tandis que le mode péristaltique devient plus efficace après un certain angle critique $\eta_{\mathrm{c}}$. Ceci peut être expliqué en considérant que la résistance de roulement réduit la capacité de traction des modes se déplaçant par roulement (modes 1 et 2). La consommation d'énergie augmente considérablement après une certaine limite qui correspond à la limite d'adhérence. L'angle critique $\eta_{\mathrm{c}}$ pour le terrain de type 2 est plus grand que pour le terrain de type 1. En effet, le terrain 2 est plus dur (la rigidité verticale équivalente $k_{\phi}+k_{\mathrm{c}} / b$ est plus grande), par conséquent le tassement $\mathrm{du}$ sol et donc la résistance au roulement sont moins importants.

\subsection{Commentaires}

L'analyse de ces résultats, nous permet d'établir quelques règles sur le choix du mode de déplacement en fonction des conditions du terrain.

Si le sol présente une faible cohésion, la capacité de traction limite le franchissement de pente. Compte tenu des résultats en terme de consommation d'énergie, le mode 3 sera donc sélectionné lorsque l'angle de pente devient supérieur à un angle critique $\eta_{\mathrm{c}}$ qui dépend des propriétés physiques du sol. Pour les angles plus faibles, le mode par roulement simple (mode 1) sera préféré.

$\mathrm{Du}$ point de vue des propriétés physiques du sol, les modes 1 et 2 sont relativement équivalents car ces deux modes génèrent un déplacement par roulement. Or, la résistance au roulement totale dépend uniquement du poids du robot et donc la distribution des efforts normaux du mode 2 n'apporte pas d'amélioration significative, sauf en présence de pente en dévers.

Par ailleurs, lorsque le sol présente d'importantes irrégularités et notamment dans le plan frontal, le mode le plus adapté est le mode roulement avec reconfiguration (mode 2). En effet, il assure la stabilité du véhicule donc son intégrité physique et de plus il permet une contribution plus homogène de la traction des quatre roues, au déplacement du robot.

\section{Conclusion}

Nous avons présenté dans cet article les différents modes de locomotion associés au robot hybride rouepatte Hylos. La modélisation du robot et de ses interactions avec l'environnement a été présentée et validée par des expériences de caractérisation du comportement du système roue-sol.

Une évaluation des modes de locomotion, s'appuyant sur des critères caractérisant l'autonomie et les performances de locomotion du système, a été développée. Cette étude permet de faire une comparaison des modes de locomotion en fonction des variations des propriétés géométriques (angle de pente) et physiques du sol. Cela conduit à établir une corrélation a priori entre les propriétés physico-géométriques de l'environnement d'exploration et le mode de locomotion approprié.

Cette étude constitue un point d'entrée au problème plus général de l'adaptation et de la supervision en ligne des modes. Cependant, la mise en place de mécanismes d'adaptation de mode nécessite des moyens de perception et d'analyse importants (stéréo-vision, analyse de texture). L'étape suivante consisterait à mettre en œuvre, à l'aide d'outils probabilistes, un sélectionneur de modes en s'appuyant sur l'extraction d'indices qualifiant le type de terrain traversé.

Il est alors nécessaire de développer des procédures d'identification en ligne des paramètres du sol. Ces procédures seront basées sur les modèles d'interaction que nous avons proposés. L'utilisation de systèmes métrologiques plus performants tels que ceux d'une centrale inertielle peuvent permettre, par fusion d'informations avec les capteurs d'effort, d'estimer la vitesse réelle du véhicule et donc les paramètres de glissement. Il est alors possible d'envisager le développement d'observateurs pour l'estimation des paramètres d'interaction. 


\section{Références}

[1] I. Leppänen, S. Salmi, A. Halme, Workpartner hutautomation's new hybrid walking machine, in Proc. of CLAWAR : Int. Conf. on Climbing and Walking Machine, 1998

[2] S. Hirose, H. Takeuchi, Study on roller-walker (basic characteristics and its control), in Proc. of ICRA : IEEE/Int. Conf. on Robotics and Automation, 1996, pp. 3265-3270

[3] F. Michaud, D. Létourneau, J.-F. Paré, M.-A. Legault, R. Cadrin, Azimut - a leg-track-wheel robot, in Proc. of IROS : IEEE/Int. Conf. on Intelligent Robots and Systems, 2003

[4] Ch. Grand, F. BenAmar, F. Plumet, Ph. Bidaud, Stability and traction optimisation of high mobility rover, The Int. J. Robotics Res., 23(10-11) (2004) 1041-1058

[5] Ch. Grand, F. BenAmar, F. Plumet, Ph. Bidaud, Decoupled posture and trajectory control of the hybrid wheel-legged robot hylos, in Proc. of ICRA : IEEE/Int. Conf. on Robotics and Automation, 2004
[6] J. Brackenbury, Fast locomotion in caterpillars, J. Insect Physiology 45 (1999) 525-533

[7] A. Grecenko, Some applications of the slip and drift theory of the wheel, in Proc. of the Int. Conf. of Society of Terrain Vehicle Systems, Detroit, 1975

[8] M.G. Bekker, Introduction to terrain-vehicle systems, The University of Michigan Press, 1969

[9] Z. Janosi, An analysis of deformable pneumatic tire performance on deformable soil, in Proc. of the 1st Int. Conf. on the Mechanics of Soil-Vehicle Systems, Torino, Italy, 1961

[10] Ch. Grand, Optimisation et commande des modes de déplacement des systèmes locomoteurs hybrides rouepatte. Application au robot Hylos, Thèse de doctorat, Université de Paris 6, 2004

[11] E. Papadopoulos, D. Rey, A new mesure of tipover stability for mobile manipulators. in Proc. of ICRA : IEEE/Int. Conf. on Robotics and Automation, 1996 pp. $3111-3116$

[12] A. Tuan Le, Modelling and control of tracked vehicles, Ph.D. thesis, University of Sydney, 1999 